\title{
Erratum: Delayed Onset of Nonthermal Melting in Single-Crystal Silicon Pumped with Hard X Rays [Phys. Rev. Lett. 120, 265701 (2018)]
}

\author{
T. Pardini, J. Alameda, A. Aquila, S. Boutet, T. Decker, A. E. Gleason, S. Guillet, P. Hamilton, M. Hayes, R. Hill, J. Koglin,
} B. Kozioziemski, J. Robinson, K. Sokolowski-Tinten, R. Soufli, and S. P. Hau-Riege

(Received 28 February 2020; accepted 4 March 2020; published 27 March 2020)

DOI: 10.1103/PhysRevLett.124.129903

A reanalysis of the crater morphology on the pillars exposed to the Linac Coherent Light Source (LCLS) beam during this experimental campaign revealed an effect we had previously overlooked. This effect is concerned with beam intensity fluctuations, and the loss of pump-probe beam overlap during one of the data runs. Unfortunately, the combined nature of these effects makes the data inconclusive as far as setting a lower limit for the time scale of the delayed onset of nonthermal melting in silicon pumped with hard $\mathrm{x}$ rays. For the reasons outlined below, we therefore retract the statement that the onset of this order-disorder transition is delayed by $150 \pm 40 \mathrm{fs}$.

In our Letter, we reported data capturing the ultrafast order-disorder transition in single crystal silicon pumped with 25 fs, $5.95 \mathrm{keV}$ x-ray pulses. The intensity of the $\mathrm{Si}(333)$ Bragg peak measured $220 \mathrm{fs}$ after pumping the system is significantly lower than that measured on unperturbed silicon, well within our measurement uncertainties. This is a clear sign of a disorder transition in progress. The femtosecond nature of the transition is in agreement with previous experimental and theoretical work on the subject.

Our data also indicated that $150 \mathrm{fs}$ after pumping the system, the $\mathrm{Si}(333)$ Bragg reflection is indistinguishable from that of unperturbed silicon. We interpreted these data as an indication that the onset of nonthermal melting must belong to the range $150<t<220 \mathrm{fs}$. With this in mind, we set a lower limit for the delayed onset of the transition at $110 \mathrm{fs}$, given our uncertainty on the delay time between pump and probe.

However, the recent discovery of beam intensity fluctuations noticeable in the probe beam during the run pertinent to the data point at $t=150 \mathrm{fs}$ forces us to retract our statement pertaining to the transition onset. A reanalysis of the size of selected craters pertaining to this run shows that craters left by the probe beam have areas consistently smaller than those in other runs by approximately a factor of 3 . Our reanalysis also revealed that, later in the same run, overlap between pump and probe beam was lost. Although it is difficult to pinpoint exactly at which point this happened, we estimate that data from $\sim 80$ shots were recorded with good overlap. If we limit our analysis to these shots only, we obtain the ratio $\left(I / I_{0}\right)=$ $0.96 \pm 0.09$ for the data point at $t=150$ fs.

Using the formula $\left(I / I_{0}\right)=\left[\left(I^{p p}+C I^{p r}\right) /\left(I_{0}^{p p}+I_{0}^{p r}\right)\right]$ described in the Letter, we derive that a ratio of 0.87 would be obtained for a complete loss of reflectivity with a pump-to-probe beam intensity ratio of $\left(I^{p p} / I^{p r}\right)=\frac{4}{1}$. The constant $C=0.35$ takes into account the area of the sample that is probed while not undergoing melting. Since, based on our reanalysis of the crater size, we cannot rule out a pump-to-probe beam intensity ratio for this run of the order of 4:1 (instead of the nominal $\sim 4: 2$ ), we conclude that our experimental data point $\left(I / I_{0}\right)=0.96 \pm 0.09$ is inconclusive as far as measuring the reflectivity of silicon $150 \mathrm{fs}$ after pumping. We therefore conclude that our data do not support our original claim setting a temporal limit for the delayed onset of nonthermal melting in silicon pumped with hard $\mathrm{x}$ rays. While it is clear that at $220 \mathrm{fs}$ after pumping the transition is under way, the experimental determination of its onset remains unknown.

At this point we can only hypothesize on the cause of the observed intensity fluctuations. We suspect that small shifts in the beam spatial position combined with the small entrance aperture of the delay line could have caused such fluctuations. Spatial shifts had been observed during the experiment, but were compensated for to the best of our ability. As for the loss of overlap during the run, we must assume it was due to some hysteresis in the pitch motor of the forth delay line mirror. However, this effect did not seem to be present in the runs corresponding to the $t=220 \mathrm{fs}$ and $t=315 \mathrm{fs}$ data points.

We conclude by pointing out an incorrect statement regarding Fig. 3. The data point at $t=385 \mathrm{fs}$ refers to a run where the beam line attenuator transmission was set to $100 \%$ instead of the nominal $14 \%$ used for the other data points. While this does not change the reported $I / I_{0}$ value, we here rectify the original statement claiming the transmission to be the same for all four data points.

This work was performed under the auspices of the U.S. Department of Energy by Lawrence Livermore National Laboratory under Contract DE-AC52-07NA27344. Document Release Number LLNL-JRNL-805472. 